\title{
Asociación sobrepeso-obesidad y tiempo de ver televisión en preescolares. Ciudad fronteriza Noreste de México
} Obesity-overweight and tv-watching time associations
in north eastern border pre-school mexican children.

Dra. Ma. de la Luz Martínez-Aguilar,• M.C.E. Pedro García-García, Dra. Rosa Ma. Aguilar-Hernández,• Dra. Laura Vázquez-Galindo,•

Dr. Gustavo Gutiérrez-Sánchez, Dr. Ricardo M. Cerda-Flores.••

\section{Resumen}

Introducción: En diversos estudios se ha encontrado asociación con el tiempo en que los niños pasan frente al televisor y la obesidad. Objetivo: Conocer la asociación de tiempo viendo la televisión y la prevalencia de sobrepeso y obesidad de niños preescolares que residen en una ciudad fronteriza de México. Método: Estudio correlacional, con124 preescolares de ambos sexos, se obtuvieron sus datos antropométricos y a los padres se les aplicó una encuesta sobre hábitos televisivos de sus hijos. Estadística descriptiva y de asociación. Resultados: El 9.8\% de los niños tenía sobrepeso y $16.1 \%$ eran obesos- El 99.2\% de ellos veían televisión. El tiempo dedicado a ver televisión fue: una a dos horas por día 75.8\%, y de tres a cuatro horas, 20.2\%. El 23.4\% de los niños tenían videojuegos. Conclusiones: La cuarta parte de los niños presentó sobrepeso y obesidad. Se encontró un mayor riesgo y asociación en niños con sobrepeso-obesidad versus niños con peso normal con: a) horas de ver televisión ( $\mathrm{OR}=2.79, \mathrm{p}=0.045)$ y b) comer cuando se ve televisión (OR $=2.87, \mathrm{p}=0.021$ ).

\section{ABstract}

Introduction: In Several studies of obesity has found association with the time that children spend watching TV. Aim: To know the association of time watching television and the prevalence of overweight and obesity in preschool children living in a border town in Mexico. Method: correlational study, 124 preschool children male and female, anthropometric data were obtained of them and their parents were surveyed about television habits of their children. It was applied descriptive statistics and of association. Results: $9.8 \%$ of children were overweight and $16.1 \%$ were obese. $99.2 \%$ of them watched television; the time spent watching television was: one to two hours per day, $75.8 \%$ and three to four hours per day, and $20.2 \% .23 .4 \%$ of children had video games. Conclusions: A quarter of children were overweight and obesity. It was found an increased risk and association in overweight and obesity children versus normal weight children with a) time of watch TV (OR $=2.79, p=$ 0.045) and b) eat when the children watch TV $(O R=2.87, p=0.021)$.

- Profesor de Tiempo Completo, Unidad Académica Multidisciplinaria Matamoros, de la Universidad Autónoma de Tamaulipas. ••Profesor de Tiempo Completo, Facultad de Enfermería. Universidad Autónoma de Nuevo León. • Correspondencia: madelaluzmartinez@yahoo.com.mx

ReCibido 23 DE Noviembre 2010. ENVIAdo 30 DE NOVIEMbre 2010. ACEPTADO 4 DE FEBRERO 2011. 


\section{INTRODUCCIÓN}

La obesidad, de acuerdo con la Organización Mundial de la Salud (OMS), es considerada como una epidemia mundial que está asociada al desarrollo de factores de riesgo tales como hiperlipidemia, hiperinsulinemia, hipertensión e intolerancia a la glucosa, así como a enfermedades crónicas como la diabetes en la vida adulta. ${ }^{1}$ Este Organismo ha catalogado a la obesidad como una enfermedad crónica, que se presenta tanto en población adulta como en población infantil. Actualmente hay en el mundo más de veintidós millones de niños menores de cinco años con obesidad o sobrepeso. ${ }^{1}$

Uno de los retos de la Salud Pública de todos los países es mantener a los niños saludables, en particular a quienes están expuestos a factores de riesgo para la salud. ${ }^{2} \mathrm{La}$ alimentación inadecuada por exceso es un factor de riesgo para la salud y conlleva a la obesidad.

La obesidad suele iniciarse en la infancia y la adolescencia por lo que constituye una enfermedad crónica, compleja, multifactorial que se puede prevenir. En estas etapas, es donde se adquieren los hábitos de alimentación y es más probable que los patrones de conducta desarrollados en la niñez y adolescencia se mantengan durante la vida adulta. ${ }^{3}$

Diversos autores han identificado a la obesidad infantil como factor de riesgo reconocido para la obesidad en el adulto. ${ }^{4-7}$

La Sociedad Latinoamericana de Nutrición, advierte que la obesidad se ha transformado en uno de los problemas más importantes que enfrentan los países, alcanzando caracteres de epidemia a lo largo de todo el ciclo vital, con sus negativos efectos sobre la salud, directamente o por su asociación con otras enfermedades crónicas no trasmisibles. ${ }^{8}$

En Chile en el 2006 el 8.1\% de los preescolares controlados en el sistema público de salud presentaban obesidad. Esta situación es mayor en los niños beneficiarios de la Junta Nacional de Jardines Infantiles (JUNJI), los cuales alcanzaron una prevalencia de $10.3 \%$, en el mismo año. ${ }^{9}$

En México, de acuerdo con la Encuesta Nacional de Salud, ${ }^{10}$ la prevalencia de sobrepeso y obesidad en los niños entre cinco y once años de edad, está en un 26.0\% para ambos sexos. Con respecto al sobrepeso en los niños, éste es de $21.2 \%$ y en las niñas de $21.8 \%$. En cuanto a la obesidad se ubicó a los hombres en $11.3 \%$ y las mujeres en $10.3 \%$.

En la zona norte del país el sobrepeso y la obesidad presentan cifras más altas $20.6 \%$ y $13.3 \%$ respectivamente para los niños de ambos sexos. ${ }^{11}$

Debido a que constituye un problema de salud pública, las tendencias de sobrepeso y obesidad en la infancia deben ser monitoreadas muy de cerca. ${ }^{12}$ En Estados Unidos se ha duplicado el número de niños que tienen exceso de peso, con respecto a hace 20 años. En Chile, México y Perú la cifra es alarmante, ya que uno de cada cuatro niños, de 4 a 10 años de edad, tiene sobrepeso o es obeso. ${ }^{13}$

El problema de la obesidad es multifactorial, entre los que se encuentra la vida sedentaria y el tiempo que pasa el niño frente al televisor.

Varios autores ${ }^{14-16}$ relacionan la obesidad con el tiempo que dedican los niños a ver televisión, o a jugar videojuegos, de ahí que se considere importante conocer la relación de las variables planteadas en esta región, con el propósito de ofrecer alternativas de solución para este problema.

En los últimos años se ha incrementado el acceso a la televisión y a los videojuegos, así como a otros medios electrónicos de distracción, ${ }^{17}$ lo cual ha modificado las formas de entretenimiento de los niños, que conducen a la disminución de la actividad física.

Sin embargo sería erróneo pensar que estos aparatos son el enemigo a vencer, y luchar por desterrarlos podría ser tan infructuoso como luchar contra la obesidad eliminando el refrigerador. ${ }^{14}$

Los niños de dos a siete años de edad de Estados Unidos pasan, en promedio, dos y media horas al día viendo televisión, videos cintas y video juegos, más aún los niños afroestadounidenses y latinos. Los niños de familias de estado socioeconómico más bajo, son los grupos demográficos con mayor riesgo de obesidad, estos tienden a ver más televisión. ${ }^{15}$

La Taylor Nelson Sofres, principal empresa que se encarga de estudios de medición de la audiencia televisiva en España, refiere que mientras en 1995, los niños entre cuatro y doce años de edad, consumieron una media de 160 minutos (dos horas y cuarenta minutos) al día ante el televisor; en el 2005 el promedio decreció a 142 minutos, lo que indica una reducción de 18 minutos en dos años. Para el 2006 se observó una reducción de dos minutos más. ${ }^{17}$

La Unión de Televisiones Comerciales Asociadas (UTECA), de España refiere que en el año 2000 
los niños de cuatro a doce años de edad también rebajan su decisión televisiva de 154 a 144 minutos, de permanecer frente al televisor. ${ }^{17}$

En un estudio realizado en Brasil $^{16}$ encontraron que los niños dedican dos horas diarias a ver televisión, conducta que origina un bajo gasto energético y que puede conducir al sedentarismo, que éste a su vez está considerado como un factor de riesgo para la obesidad. En México, en el estado de San Luis Potosí, Moreno y Toro ${ }^{18}$ realizaron un estudio donde encontraron que el $41 \%$ de los niños estudiados veía televisión de una a tres horas, pero un 29\% pasa más de tres horas diarias frente al televisor.

Además de la televisión, el uso de la computadora y los videojuegos han acaparado la atención de los niños, en detrimento de la salud, ya que el tiempo destinado al uso de estos equipos restringe el que debe dedicarse al ejercicio y a tener otras actividades. ${ }^{15}$

De acuerdo con Zuawadzki, ${ }^{14}$ el niño promedio ve más de 20,000 comerciales por año, $60 \%$ de los cuales promueven cereales, dulces, alimentos inadecuados y juguetes.

Zuawadzki, ${ }^{14}$ refiere que los neurocientíficos han demostrado que la exposición repetida de un estímulo procedente del entorno del niño forzosamente hará impacto en su desarrollo mental y emocional. Así las técnicas subliminales utilizadas por la televisión pueden producir en un cerebro en desarrollo pérdida de la capacidad de razonamiento independiente, necesaria para actividades como el juego, manualidades, interacción social o para correr bulliciosamente. En cuanto al gusto por ver televisión, el $97 \%$ de los infantes les gusta y un
$81 \%$ comen cuando la ven. ${ }^{18}$

Castelli ${ }^{19}$ relata que el entretenimiento no debe ir acompañado del consumo de calorías. Hay dos consecuencias de comer y ver televisión, por una parte propicia la vida sedentaria y por otro el niño recibe información de alimentos de bajo contenido nutricional y de alto contenido calórico, ${ }^{20,15}$ lo cual conlleva al sobrepeso y a la obesidad.

Veerman y cols, ${ }^{21}$ en un estudio, refieren que la prohibición total de publicidad de alimentos en la televisión puede reducir la prevalencia de obesidad en los niños de Estados Unidos.

Otro aspecto importante es con quien ven la televisión los niños. Lazo y Gabelas ${ }^{17}$ reportan que el $51.3 \%$ de los niños ven la televisión con sus padres, el $38.0 \%$ con sus hermanos. Ya que es de suma importancia, que el niño vea la televisión con un adulto y éste establezca dialogo respecto al tema visto y así incrementar la capacidad crítica y autónoma de los niños respecto al material que se les presenta.

Moreno y Toro $^{18}$ refieren que los niños ven televisión sentados o acostados en la sala y el $43 \%$ acostados en un cuarto, lo cual disminuye a su mínima expresión cualquier actividad física donde pueda tener un gasto energético, y consuma las calorías que ingresaron al organismo.

La American Academy of Pediatrics ${ }^{22}$ refiere que los niños que ven televisión más de cuatro horas por día tenían un Índice de Masa Corporal (IMC) mayor comparado con el de los niños que ven televisión menos de dos horas por día. Así como el tener un televisor en la recamara es un fuerte pronosticador de tener sobrepeso, aún en los niños preescolares. No sorprende encontrar que los niños que pasan mayor tiempo frente al televisor presenten sobrepeso y obesidad.

Considerando que las horas que pasa el niño viendo televisión o jugando videojuegos pueden ser un factor predisponente para el sobrepeso y la obesidad, se decidió desarrollar el presente trabajo de investigación con el objetivo de conocer en niños preescolares residentes de una ciudad fronteriza de México, la prevalencia de sobrepeso y obesidad y su asociación con el tiempo de ver televisión.

Se pretende que el resultado de este estudio aporte conocimiento para la toma de decisiones del equipo multidisciplinario de salud, donde la enfermera juega un papel preponderante; para incidir en la educación para la salud que incluye modificación de hábitos de alimentación y de conductas nocivas, como son el exceso del tiempo de ver televisión y disminución del tiempo para el ejercicio y el juego.

\section{Metodología}

Estudio correlacional en un Jardín de Niños de una comunidad urbano marginada de Matamoros, Tamaulipas, México, en el año 2010. La población estuvo conformada por 124 alumnos y debido a su extensión fue posible realizar las mediciones en todos los niños. Estos fueron de ambos sexos, entre 4 y 6 años de edad, de segundo y tercer grado. La recolección de la información fue realizada por pasantes de licenciatura en enfermería, las cuales fueron capacitadas para realizar los procedimientos. La técnica utilizada fue la antropometría, mediante la cual se obtuvieron los datos de los niños y se registraron 
en un formato diseñado ex profeso. Se aplicó una encuesta a los padres de familia con siete preguntas cerradas, de opción múltiple, para explorar hábitos del niño en cuanto a ver televisión, tipo de programación, jugar con videojuegos, y ver televisión y comer.

Con los datos antropométricos se calculó el IMC y se registró en percentiles, posteriormente se clasificó por edad y sexo, de acuerdo con lo establecido por el Center for Disease Control and Preventión CDC $(2000)^{23}$ en: Obesidad (percentil $\geq 95$ ), Sobrepeso (percentil $\geq$ 85 pero $<95$ ), peso normal (percentil $>5$ pero $<85)$ y bajo peso (percentil $\leq 5$ ).

Los datos obtenidos fueron capturados y analizados con el programa estadístico SPSS Versión 17. Se calcularon estadísticas descriptivas y se analizó la asociación mediante el paquete RXC de Miller con 50,000 simulacros, esta prueba se utiliza en aquellos casos donde el número de individuos por celda es menor de cinco. Y la razón de momios (OR) fue obtenida mediante la calculadora online EpiMax.

De acuerdo con el Reglamento de la Ley General de Salud en materia de Investigación, ${ }^{24}$ el presente estudio se considera sin riesgo de daño físico o psicológico ya que no hay invasión corporal.

\section{Resultados}

Con respecto al sexo en la muestra estudiada, predominó el masculino con el $51.6 \%$ y el femenino con 48.3 $\%$. En cuanto a la edad, prevalecieron los niños de cinco años con un $54.0 \%$. En cuanto al estado nutricional, se encontró peso normal en el $63.8 \%$, obesidad en el $16.1 \%$, sobrepeso en el $9.8 \%$ y bajo peso en el
10.4\%. Se encontró que el $25.8 \%$ de los niños de ambos sexos presenta problemas de sobrepeso y obesidad. Estos datos distribuidos por edad son: $15.4 \%$ en los niños de cinco años, $8.1 \%$ en los de seis años y el $2.4 \%$ en los de cuatro años.

En relación con los hábitos de ver televisión, el 99.2\% de las madres de familia refieren que su hijo ve televisión. En cuanto al tiempo dedicado a ver televisión, el 75.8\% de una a dos horas, el $20.2 \%$ de 3 a 4 horas y el $4.0 \%$ dice que la ve entre cinco y seis horas. Los programas preferidos de los niños son: el $76.6 \%$ caricaturas, $11.3 \%$ series, $8.9 \%$ programas educativos y $3.2 \%$ refiere que ven novelas. El 23.4\% de los niños cuenta con un videojuego, de los cuales el 17.7\% dedica de una a dos horas diarias, el $4.8 \%$ de tres a cuatro horas y el $0.8 \%$ juega entre cinco y seis horas. El 47.6\% refirió comer cuando ve televisión.

Se observó una relación significativa entre las variables comer cuando ve televisión y peso $\mathrm{p}=$ (0.021), con un riesgo 2.87 veces mayor en los niños con sobrepeso y obesidad. También resultó significativa la relación de horas dedicadas a ver televisión por los niños con sobrepeso y obesidad $\mathrm{p}=(0.045)$ con un riesgo de 2.79 veces mayor, que en niños con peso normal.

\section{Discusión}

En este estudio el mayor porcentaje de los niños ven televisión de una a dos horas diarias, el tiempo es ligeramente menor que lo reportado por Reyes y cols ${ }^{15}$ en Estados Unidos y por la Taylor Nelson Sofres de España ${ }^{17}$ y en Brasil según Borges, et al. ${ }^{16}$ Sin embargo los resultados obtenidos son similares a lo reportado por Anderson ${ }^{25}$ y Zim- merman. ${ }^{26}$

En relación con las horas dedicadas por el niño a jugar con videojuegos el resultado del presente estudio es similar a lo reportado por Reyes y cols. ${ }^{15}$

Se encontró similitud en los datos de sobrepeso y obesidad de este estudio con los reportados por ENSANUT 2006. ${ }^{10}$

También el porcentaje del sobrepeso encontrado es menor a lo referido para el estado de Tamaulipas por la ENSANUT 2006 resultados en las fronteras. ${ }^{11}$

En el presente estudio se observó asociación entre las variables sobrepeso y obesidad y comer cuando se ve la televisión, en cambio Moraes $^{27}$ en su estudio refiere no haberla encontrado.

\section{Conclusiones}

La cuarta parte de los niños estudiados presentó sobrepeso y obesidad.

Se encontró una relación significativa entre las variables comer cuando ve televisión, así como horas dedicadas a ver televisión y el peso de los preescolares.

La infancia es la etapa donde se adquieren los hábitos de alimentación y actividad física que contribuyen a una vida saludable. Por lo que es importante poner atención en su fomento en esta fase de vida, para evitar el sobrepeso y la obesidad. En esta etapa ha sido un factor contribuyente para estos problemas el exceso en el tiempo dedicado a ver televisión y jugar videojuegos.

Por lo que se recomienda al personal sanitario, ofrecer educación para la salud a los padres de familia para concientizarlos sobre el problema y que éstos a su vez motiven a sus hijos a realzar algún tipo de actividad física y disminuir el tiempo dedicado a ver la televisión. 
Tabla 1: Distribución del estado nutrición en función de ocho preguntas aplicadas

\begin{tabular}{|c|c|c|c|c|}
\hline \multirow[b]{3}{*}{ Pregunta } & \multirow[b]{3}{*}{ Respuesta } & \multicolumn{2}{|c|}{ Estado Nutricio } & \multirow[b]{2}{*}{ Prob* } \\
\hline & & Normal & SP-OB & \\
\hline & & $n=79$ & $\mathrm{n}=32$ & \\
\hline \multirow[t]{2}{*}{ ¿Su niño ve televisión? } & $\mathrm{Si}$ & 7898.73 & 32100.00 & 1.000 \\
\hline & No & 11.27 & $0 \quad 0.00$ & \\
\hline \multirow{2}{*}{$\begin{array}{l}\text { ¿Cuántas horas dedica al día } \\
\text { para ver la televisión? }\end{array}$} & $1-2$ & $65 \quad 82.28$ & $20 \quad 62.50$ & $0.045^{* * *}$ \\
\hline & $>2$ & $14 \quad 17.72$ & 1237.50 & \\
\hline \multirow{4}{*}{$\begin{array}{l}\text { ¿Qué programas son los que } \\
\text { más le gustan? }\end{array}$} & Caricaturas & $64 \quad 81.01$ & 2268.75 & 0.424 \\
\hline & Series & 67.59 & 515.63 & \\
\hline & Novelas & 22.53 & 13.12 & \\
\hline & Programas educativos & 78.86 & $4 \quad 12.50$ & \\
\hline \multirow[t]{2}{*}{ ¿Su hijo ve caricaturas? } & $\mathrm{Si}$ & 6481.01 & 2268.75 & 0.200 \\
\hline & No & $15 \quad 18.99$ & 1031.25 & \\
\hline \multirow[t]{3}{*}{ ¿Su hijo tiene algún videojuego? } & $\mathrm{Si}$ & $16 \quad 20.25$ & 825.00 & 0.608 \\
\hline & No & 6379.75 & $24 \quad 75.00$ & \\
\hline & No tiene & 6379.75 & $24 \quad 75.00$ & 0.692 \\
\hline \multirow{3}{*}{$\begin{array}{l}\text { ¿Cuántas horas al día juega } \\
\text { con el videojuego }\end{array}$} & $1-2$ & 1215.19 & 515.62 & \\
\hline & $3-4$ & 33.80 & 39.38 & \\
\hline & $5-6$ & 11.26 & $0 \quad 0.00$ & \\
\hline \multirow{2}{*}{$\begin{array}{l}\text { ¿Su hijo acostumbra a comer } \\
\text { cuando ve la televisión? }\end{array}$} & $\mathrm{Si}$ & 2936.71 & $20 \quad 62.50$ & $0.021^{* * *}$ \\
\hline & No & $\begin{array}{ll}50 & 63.29\end{array}$ & $12 \quad 37.50$ & \\
\hline \multirow{2}{*}{$\begin{array}{l}\text { ¿Algún miembro de la familia } \\
\text { padece obesidad? }\end{array}$} & $\mathrm{Si}$ & 2126.58 & $12 \quad 37.50$ & 0.247 \\
\hline & No & $58 \quad 73.42$ & $20 \quad 62.50$ & \\
\hline
\end{tabular}

\section{Referencias Bibliográficas}

1. Organización Mundial de la Salud.

Comunicado de prensa 11 noviembre 2004. [Recuperado 10
Enero 2011]. Disponible en: http:// bit.ly/po01N3.

2. Santos P, Villa BJ, García AM, León

AG, Quezada BS, Tapia CR. La transición epidemiológica de las y los adolescentes en México. Salud Pública Mex 2003;1(45):14052. 
3. Shepherd J, Harden A, Rees R, Brunton G, Garcia J, Oliver S, Oakley, A. Young people and healthy eating: a systematic review of research on barriers and facilitators. Health Education Research.2006; 21 (2), 239-57.

4. Barrio R, López M, Colino E, Mustieles C, Alonso M. Obesidad y síndrome metabólico en la infancia. Endocrinología y Nutrición 2005; 52(02): 65- 74.

5. Dalmau J, Alonso M, Gómez L, Martínez C, Sierra C. Obesidad Infantil. Recomendaciones del Comité de Nutrición de la Asociación Española de Pediatría. Parte II. Diagnóstico. Comorbilidades. Tratamiento. An Pediatr (Barc). 2007; 66(3):294-304

6. Writ $\mathrm{CH}$, Parker L, Lamont D, Craft A. Implications of childhood obesity for adult health: findings from thousand families cohort study. BMJ.2001; 323: 1280-4.

7. Law C. Adult obesity and growth in childhood. BMJ.2001; 323: 132021.

8. Atalah SE. La Sociedad Latinoamericana de Nutrición, SLAN (20062009). ALAN. 2007; 57 (2): 107-107.

9. Vázquez F, Andrade M, Rodríguez M P \& Salazar G. Efecto de un programa de educación nutricional en el consumo de energía y macronutrientes de preescolares asistentes a jardines infantiles Junji de zona oriente de Santiago, Chile. Archivos Latinoamericanos de Nutrición 2008; 58(3): 241-8.

10.Olaiz-Fernández G, Rivera-Dommarco J, Shamah-Levy T, Rojas R, Villalpando-Hernández $\mathrm{S}$, Hernández-Avila M, SepúlvedaAmor J. Encuesta Nacional de Salud y Nutrición 2006. Cuernavaca, México: Instituto Nacional de Salud Pública, 2006: 89-95.
11. Instituto Nacional de Salud Pública. Encuesta Nacional de Salud y Nutrición 2006. Resultados en las Fronteras. Cuernavaca, México: Instituto Nacional de Salud PúblicaSecretaría de Salud, 2008: 119-134.

12. Oude Luttikhuis H, Baur L, Jansen H, Shrewsbury VA, O'Malley C, Stolk RP, Summerbell CD Interventions for treating obesity in children. Cochrane Database of Systematic Reviews 2009, Issue 1. Art. No.: CD001872. DOI: 10.1002/14651858.CD001872.pub2

13. Eberwine D, Obesidad: el gran desafío, Perspectivas de la salud OPS, 2002; 7 (3).Disponible en: http://bit.ly/oSe1Lk

14. Zuawadzki N. Apague el televisor, no su hijo. Pediatría. 2002; 29 (1). Disponible en: http://bit.ly/ nybmTK

15. Reyes U, Sánchez NP, Reyes U, Reyes D, Carbajal L, La televisión y los niños: II Obesidad. Boletín Clínico Hospital Infantil del Estado de Sonora. 2006; 23 (1): 25-30.

16. Borges C, Kraft ML, Lima M, Fernández $\mathrm{A}$, Teramoto $\mathrm{A}$, Kanunfre C. Influencia da televisao na prevalencia de obesidade infantil em Ponta Grossa, Paraná. Ciencia Cuid Saude. 2007; 6 (3):305-11.

17. Marta C, Gabelas J. La televisión: epicentro de la convergencia entre pantallas. Enlace: Revista Venezolana de Información Tecnológica y Conocimiento 2008; 5 (1): 11-23.

18. Moreno A, Toro L. La televisión, mediadora entre consumismo y obesidad. Rev Chil Nutr 2009; 36(1): 46-52.

19. Castelli D. La obesidad infantil. septiembre de 2006. \{Consultado el 5 de octubre de 2010.\}. Disponible en: http://bit.ly/pbDd2H

20. Powell LM, Szczypka G, Chaloupka FJ, Braunschweig CL Nutritional content of television food advertisements seen by children and adolescents in the United States. Pediatrics. 2007; 120(3):576-83

21. Veerman JL, Van Beeck EF, Barendregt JJ, Mackenbach JP. By how much would limiting TV food advertising reduce childhood obesity?. Eur J Public Health. 2009; 19 (4): 365-9.

22. American Academy of Pediatrics. Committee on Nutrition. Prevention of Pediatric Overweight and Obesity. Pediatrics. 2003; 112; 424-30.

23. Center for Disease Control and Prevention. (2000). Percentiles de estatura por edad y sexo. \{Consultado el 15 de octubre de 2010\}. Disponible en: http://www.cdc. gov/growthcharts.

24. Secretaría de Salud. Reglamento de la Ley General de Salud en materia de Investigación para la salud. 1987.

25. Anderson S E, Economos C D, Must A. Active Play and Screen Time in US Children Aged 4 to 11 Years in Relation to Sociodemographic and Weight Status Characteristics: A Nationally Representative Crosssectional Analysis. BMC Public Health. 2008, 8:366.

26. Zimmerman FJ, Bell JF. Associations of Television Content Type and Obesity in children. Am J Public Health. 2010;100(2):334-40.

27. Moraes AS, Beltran RJ, Mondini L, Martins I C.Prevalencia of overweight and obesity, and associated factors in school children from urban area in Chilpancingo Guerrero, México. Cad. Saude Publica. 2006; 22 (6): 1289-301. 